\title{
NORMS FOR COPULAS
}

\section{WILLIAM F. DARSOW and ELWOOD T. OLSEN}

Department of Mathematics

Illinois Institute of Technology

Chicago, Illinois 60616

(Received October 19, 1993 and in revised form July 20, 1994)

\begin{abstract}
We consider several norms on the span of the set $\mathcal{C}$ of all copulas. Dominance and equivalence relationships among the norms are discussed, and completeness issues are addressed. The motivation for the study is discussed. Applications to the study of one parameter semigroups of copulas are also addressed.
\end{abstract}

KEY WORDS AND PHRASES. Copulas, doubly stochastic measures, Banach algebras, one parameter semigroups.

1991 AMS SUBJECT CLASSIFICATION CODES. 46B20, 28A35, 60J25

\section{INTRODUCTION.}

A copula is a function $C:[0,1]^{2} \rightarrow[0,1]$ satisfying the boundary conditions $C(x, 0)=$ $C(0, y)=0, C(x, 1)=x$ and $C(1, y)=y$, for all $x, y \in[0,1]$, and the monotonicity condition

$$
C\left(x_{1}, y_{1}\right)-C\left(x_{2}, y_{1}\right)-C\left(x_{1}, y_{2}\right)+C\left(x_{2}, y_{2}\right) \geq 0
$$

whenever $x_{1} \leq x_{2}$ and $y_{1} \leq y_{2}$. These conditions imply that $C$ is a continuous function. Copulas are of interest because they link joint distributions to marginal distributions: for any real valued random variables $X_{1}$ and $X_{2}$ with joint distribution $F_{12}$ there is a copula $C$ such that

$$
F_{12}\left(x_{1}, x_{2}\right)=C\left(F_{1}\left(x_{1}\right), F_{2}\left(x_{2}\right)\right)
$$

where $F_{1}$ and $F_{2}$ denote the cumulative distribution functions of $X_{1}$ and $X_{2}$, respectively. For a proof, see Sklar[6, 7]. Knowledge of basic properties of copulas will be assumed in this paper, though references will often be given when they are used. For a discussion of these properties, see Darsow et al. [2]; Sklar [6, 7].

We denote by $\mathcal{C}$ the set of all copulas. $\mathcal{C}$ is closed under convex combinations and under a product operation $A * B$ defined by

$$
A * B(x, y)=\int_{0}^{1} A_{, 2}(x, t) B, 1(t, y) d t .
$$

Here and in the sequel the subscripts , 1 and , 2 denote partial derivatives:

$$
B_{, 1}(x, y)=\frac{\partial B}{\partial x}(x, y) \quad A_{, 2}(x, y)=\frac{\partial A}{\partial y}(x, y) .
$$


The copulas $P(x, y)=x y$ and $M(x, y)=\min \{x, y\}$ are null and unit elements with respect to the product operation; that is,

$$
A * P=P * A=P \quad A * M=M * A=A
$$

for all copulas $A$, as is readily verified using the definition of the product. A copula $E$ is idempotent if $E * E=E$; both $P$ and $M$ are idempotent, and there are many other idempotents. A copula $A$ is left invertible if there is a copula $B$ such that $B * A=M$ and right invertible if there is a copula $B$ such that $A * B=M$. If $A$ is left invertible, its left inverse is the copula $A^{T}$ defined by $A^{T}(x, y)=A(y, x)$; similarly, if $A$ is right invertible, its right inverse is $A^{T}$, Darsow et al. [2]. There exist copulas which possess one-sided but not two-sided inverses.

The set $\mathcal{C}$ is also closed under uniform limits: if $A_{n} \in \mathcal{C}$ for all $n$ and $A_{n} \rightarrow A$ uniformly, then necessarily $A \in \mathcal{C}$. In fact, the set $\mathcal{C}$ is a compact subset of $C^{0}\left([0,1]^{2}\right)$ under the uniform norm. This is a useful property. However, the product does not behave well under the uniform norm. If $A_{n} \rightarrow A$ uniformly and $B$ is any copula, then $A_{n} * B \rightarrow A * B$ and $B * A_{n} \rightarrow B * A$, Darsow et al. [2], so that the product is continuous in each place. But the product is not jointly continuous. It can be shown that (two-sided) invertible copulas are dense in $\mathcal{C}$ in the topology of uniform convergence. If $A_{n}$ is a sequence of invertible copulas converging to a noninvertible copula, say $P$, then $A_{n}^{T} * A_{n}=M$ for all $n$ but $P^{T} * P=P \neq M$. It follows that the product is not jointly continuous. This is an inconvenient fact, and a part of the motivation for the research reported here was to find a natural topology on the set of copulas under which the product operation was not only continuous in each place but also jointly continuous. The joint continuity issue is discussed at various points in the paper.

One parameter semigroups of copulas arise naturally in the study of Markov processes, Darsow et al. [2]. Let $C$ be any copula, let $E$ be an idempotent copula satisfying $C * E=E * C=C$, and let $a$ be a positive number. Then $A_{t}$ defined for $t \geq 0$ by

$$
A_{t}=e^{-a t}\left(E+\sum_{k=1}^{\infty} \frac{(a t)^{k}}{k !} C^{k}\right)
$$

is a semigroup of copulas: $A_{s+t}=A_{s} * A_{t}$. In the definition, $C^{k}$ denotes the $k$-fold $*$ product of $C$ with itself; the series converges uniformly for all $a$ and $t$, as is easy to verify. The classical theory of one parameter semigroups is developed in the context of Banach algebras. To investigate the applicability of the classical theory to semigroups of copulas, it is natural, first, to work in the span of $\mathcal{C}$, which we will denote span $(\mathcal{C})$, rather than $\mathcal{C}$ itself, and, second, to seek a norm under which span $(\mathcal{C})$ is in fact a Banach algebra. This was a second part of the motivation for the research reported here. The application of this research to one parameter semigroups of copulas is discussed in Section 6 of this paper.

The paper is organized as follows: In Section 2, Preliminaries, we discuss span $(\mathcal{C})$ and define the norms to be treated in subsequent sections. In Sections 3, 4 and 5, we discuss the Minkowski norm, Sobolev norms, and the Jordan norm on span $(\mathcal{C})$, respectively. Section 6 is devoted to semigroups of copulas and Section 7, to discussion and conclusions; in particular, we shall make some comments about compactness in Section 7.

\section{PRELIMINARIES.}

By span $(\mathcal{C})$ we mean the linear span of the copulas - the set of all finite real linear combinations of elements of $\mathcal{C}$. Any element $A \in \operatorname{span}(\mathcal{C})$ can be written in the form

$$
A=s B-t C
$$

where $s$ and $t$ are nonnegative and $B$ and $C$ are copulas. To see this, observe that if

$$
A=\sum_{k=1}^{n} a_{k} A_{k}
$$


where $A_{k} \in \mathcal{C}$ and $a_{k}$ is a real number, then we can write

$$
A=s \sum_{a_{k}>0} \frac{a_{k}}{s} A_{k}-t \sum_{a_{k}<0} \frac{-a_{k}}{t} A_{k}=s B-t C
$$

where the last equality defines $B$ and $C$ and

$$
s=\sum_{a_{k}>0} a_{k} \quad \text { and } \quad t=-\sum_{a_{k}<0} a_{k} .
$$

(In both of the foregoing equations, the sum is taken to be zero if the set summed over is empty.) Observe that $B$ and $C$ are copulas, since $\mathcal{C}$ is closed under convex combinations. We will make repeated use of the observation (2.1), usually without comment.

We now define the norms we will investigate.

Minkowski norm. The symbol $\mathcal{B}=\operatorname{co}(-\mathcal{C} \cup \mathcal{C})$ denotes the convex hull of the set $-\mathcal{C} \cup \mathcal{C}$, that is, the set of all convex combinations of members of $\mathcal{C}$ and $-\mathcal{C}$. It is easy to see that $\operatorname{span}(\mathcal{C})=\cup_{t \geq 0} t \mathcal{B}$. For any $A \in \operatorname{span}(\mathcal{C})$ define

$$
\|A\|_{M}=\inf \{t>0 \mid A \in t \mathcal{B}\}
$$

$\|\cdot\|_{M}$ is sometimes referred to as a Minkowski functional; it is easy to verify that it is a norm on span $(\mathcal{C})$, e.g. Rudin [5]. There is a more convenient definition of the Minkowski functional:

$$
\|A\|_{M}=\inf \{s+t \mid s, t \geq 0 \text { and } A=s B-t C \text { for some } B, C \in \mathcal{C}\}
$$

It is easy to verify that (2.2) and (2.3) are equivalent definitions; we omit the proof.

We will show in Section 3 that span $(\mathcal{C})$ is a Banach algebra under the norm $\|\cdot\|_{M}$. The problem with the Minkowski functional is that it is difficult to compute and thus is cumbersome to work with. We include some results in Section 3 which facilitate the calculation of the Minkowski norm for certain special classes of elements of span $(\mathcal{C})$.

Sobolev norms. Since the $*$ product is defined using first partial derivatives, it seems natural to work with norms which take derivatives into account. It is readily verified that

$$
\mathcal{C} \subset W^{m, p}\left([0,1]^{2}\right)
$$

for $m=0,1$ and all $p \in[1, \infty] . W^{m, p}(\Omega)$ denotes the Sobolev space

$$
\left\{f \mid f \in L^{p}(\Omega) \text { and } D^{\alpha} f \in L^{p}(\Omega) \text { for all multi-indices } \alpha \text { for which }|\alpha| \leq m\right\} \text {. }
$$

Here, $\Omega \subset R^{d}$, a multi-index $\alpha$ is a $d$-tuple of nonnegative integers, $|\alpha|$ denotes the sum of the components of $\alpha$, and $D^{\alpha}$ denotes a distributional partial derivative. In our case, $d=2$ and $m=0$ or 1 , so that $\alpha=(0,0),(1,0)$ or $(0,1)$, and $D^{(0,0)} f=f, D^{(1,0)} f=f_{, 1}$ and $D^{(0,1)} f=f_{, 2}$. It is well known, e.g. Adams [1], that each of the Sobolev spaces so defined is a Banach space (i.e., is complete) under the norm defined by

$$
\|f\|_{m, p}= \begin{cases}\left(\sum_{0 \leq|\alpha| \leq m}\left\|D^{\alpha} f\right\|_{p}^{p}\right)^{1 / p} & p \in[1, \infty) \\ \max _{0 \leq|\alpha| \leq m}\left\|D^{\alpha} f\right\|_{\infty} & p=\infty\end{cases}
$$

We will show in Section 4 that $\mathcal{C}$ is a complete metric space under the Sobolev norms $\|\cdot\|_{1, p}$ and that on $\mathcal{C}$ the product is jointly continuous with respect to $\|\cdot\|_{1, p}$ for $p \in[1, \infty)$; it is an open issue whether the product is continuous (jointly or separately) with respect to the norm $\|\cdot\|_{1, \infty}$. We will also show that, on $\operatorname{span}(\mathcal{C})$ each of the Sobolev norms is dominated by, but is not equivalent to, the Minkowski functional $\|\cdot\|_{M}$ and that span $(\mathcal{C})$ is not a closed subspace of 
any of the $W^{m, p}$ spaces. The issue of continuity of the * product on span $(\mathcal{C})$, as opposed to $\mathcal{C}$ itself, is an open one.

Jordan norm. We have occasionally found it convenient to work with one other norm.

It is well known, cf. Darsow et al. [2], that a copula induces a unique doubly stochastic probability measure $\mu_{C}$ on $[0,1]^{2}$ via the assignment

$$
\left.\mu_{C}\left(\mid x_{1}, x_{2}\right] \times\left[y_{1}, y_{2}\right]\right)=C\left(x_{1}, y_{1}\right)-C\left(x_{2}, y_{1}\right)-C\left(x_{1}, y_{2}\right)+C\left(x_{2}, y_{2}\right)
$$

as the measure of a rectangle. Conversely, for any doubly stochastic measure $\mu$, there is a unique copula $C_{\mu}$ defined via

$$
C_{\mu}(x, y)=\mu([0, x] \times[0, y]) .
$$

Thus, doubly stochastic measures on $[0,1]^{2}$ and copulas are in one to one correspondence. An element $C \in \operatorname{span}(\mathcal{C})$ induces a finite signed measure on $[0,1]^{2}$ via the definition (2.5). All linear combinations of doubly stochastic measures are obtained in this manner.

It is also well known, cf. Halmos [3], that for any finite signed measure $\mu$ there exist measurable sets $E^{+}$and $E^{-}$such that $E^{+} \cap E^{-}=\phi, E^{+} \cup E^{-}=[0,1]^{2}$ and, for all measurable sets $F, \mu\left(E^{+} \cap F\right) \geq 0$ and $\mu\left(E^{-} \cap F\right) \leq 0$. It follows that $\mu^{+}$and $\mu^{-}$defined by

$$
\mu^{+}(F)=\mu\left(E^{+} \cap F\right) \quad \mu^{-}(F)=-\mu\left(E^{-} \cap F\right)
$$

are measures. Furthermore, $\mu^{+}$and $\mu^{-}$are unique, though the sets $E^{+}$and $E^{-}$need not be. The decomposition $\mu=\mu^{+}-\mu^{-}$of a finite signed measure as the difference of two measures is called the Jordan decomposition. The set of finite signed measures is closed under real linear combinations, and it is an easy exercise to show that

$$
\|\mu\|_{J}=\mu^{+}\left([0,1]^{2}\right)+\mu^{-}\left([0,1]^{2}\right)
$$

defines a norm on the set under which the set is a Banach space, Halmos [3, p. 123, Ex. (4)].

For $A \in \operatorname{span}(\mathcal{C})$ we define the "Jordan" (or total variation) norm

$$
\|A\|_{J}=\left\|\mu_{A}\right\|_{J}
$$

We will show in Section 5 that the Jordan norm dominates, but is not equivalent to, the uniform norm and is dominated by, but is not equivalent to, the Minkowski functional. We will also show that span $(\mathcal{C})$ is not complete with respect to the Jordan norm. The continuity (or lack thereof) of the * product in the topology of the Jordan norm is an open issue.

\section{MINKOWSKI NORM.}

We will show that span $(\mathcal{C})$ is a Banach algebra under the Minkowski norm. The proof will be by way of two preliminary lemmas and two theorems. Then we will state and prove some miscellaneous facts which are useful in computing the Minkowski norm of an element of span $(\mathcal{C})$ in some special cases.

LEMMA 3.1. The Minkowski norm dominates the uniform $\left(W^{0, \infty}\right)$ norm on span $(\mathcal{C})$.

PROOF. Observe that if $A=s B-t C$, where $s, t \geq 0$ and $B, C \in \mathcal{C}$, then

$$
\|A\|_{0, \infty} \leq s\|B\|_{0, \infty}+t\|C\|_{0, \infty}=s+t
$$

since the uniform norm of any copula is 1 . Thus, $\|A\|_{0, \infty}$ is a lower bound of the set whose greatest lower bound is the Minkowski norm. The desired conclusion follows.

The infimum in the definition (2.2) of the Minkowski norm is actually achieved:

LEMMA 3.2. For any element $A \in \operatorname{span}(\mathcal{C})$, there exist numbers $s, t \geq 0$ and $B, C \in \mathcal{C}$ such that $A=s B-t C$ and $\|A\|_{M}=s+t$. 
PROOF. By the property of the infimum, there exist numbers $s_{n}, t_{n} \geq 0$ and copulas $B_{n}, C_{n}$ such that $A=s_{n} B_{n}-t_{n} C_{n}$ and $s_{n}+t_{n} \downarrow\|A\|_{M}$. But $B_{n}(1,1)=C_{n}(1,1)=1$ for all $n$, so $s_{n}-t_{n}=A(1,1)$ is a constant (and thus convergent) sequence. It follows that the sequences $s_{n}$ and $t_{n}$ both converge; call their limits $s$ and $t$. Furthermore, since $\mathcal{C}$ is compact in the uniform topology, there is an increasing sequence of indices $n_{k}$ such that $B_{n_{k}}$ and $C_{n_{k}}$ both converge uniformly; call their uniform limits $B$ and $C$. Clearly $A=s B-t C$. \

THEOREM 3.1. $\operatorname{Span}(\mathcal{C})$ is complete under $\|\cdot\|_{M}$.

PROOF. This is a classical proof, adapted to the case of copulas. Suppose that $A_{n}$ is Cauchy with respect to $\|\cdot\|_{M}$. Passing to a subsequence, if need be, assume that $\left\|A_{n+1}-A_{n}\right\|_{M} \leq 2^{-(n+1)}$ for all $n$. Using Lemma 3.2, we can find nonnegative numbers $s_{n}$ and $t_{n}$ and copulas $B_{n}$ and $C_{n}$ such that

$$
A_{n}=s_{n} B_{n}-t_{n} C_{n} \quad \text { and } \quad s_{n}+t_{n}=\left\|A_{n}\right\|_{M} .
$$

Observe that (by the triangle inequality) the sequence of numbers $\left\|A_{n}\right\|_{M}=s_{n}+t_{n}$ is Cauchy, and observe also that $s_{n}-t_{n}=A_{n}(1,1)$ is Cauchy, since by Lemma 3.1 the Minkowski norm dominates the uniform norm. It follows upon taking sums and differences that both $s_{n}$ and $t_{n}$ are Cauchy; call their limits $s$ and $t$. Since $\mathcal{C}$ is compact, we can find an increasing sequence $n_{k}$ of indices such that $B_{n_{k}}$ and $C_{n_{k}}$ both converge uniformly; call their limits $B$ and $C$. Set $A=s B-t C$ and observe that $A \in \operatorname{span}(\mathcal{C})$. Clearly $A_{n_{k}} \rightarrow A$ uniformly, and $A_{n} \rightarrow A$ uniformly follows easily. To complete the proof, observe that we can write

$$
A=A_{1}+\sum_{k=1}^{\infty}\left(A_{k+1}-A_{k}\right) .
$$

This is true pointwise, by the argument above. But also

$$
\begin{aligned}
\left\|A-A_{n}\right\|_{M} & =\left\|\sum_{k=n}^{\infty}\left(A_{k+1}-A_{k}\right)\right\|_{M} \\
& \leq \sum_{k=n}^{\infty} 2^{-(k+1)} .
\end{aligned}
$$

Thus, $\left\|A-A_{n}\right\|_{M} \rightarrow 0$, and span $(\mathcal{C})$ is complete under $\|\cdot\|_{M}$.

The following theorem completes the proof that span $(\mathcal{C})$, together with the $*$ product, is a Banach algebra. It is not obvious, but $*$ defines an associative binary operation on $\mathcal{C}$, Darsow et al. [2], and associativity for the extension of $*$ to all of span $(\mathcal{C})$ follows, so we need not address this issue.

THEOREM 3.2. For all $A_{1}, A_{2} \in \operatorname{span}(\mathcal{C}),\left\|A_{1} * A_{2}\right\|_{M} \leq\left\|A_{1}\right\|_{M}\left\|A_{2}\right\|_{M}$.

PROOF. Write $A_{k}=s_{k} B_{k}-t_{k} C_{k}$ where $s_{k}, t_{k} \geq 0, B_{k}, C_{k} \in \mathcal{C}$ and $s_{k}+t_{k}=\left\|A_{k}\right\|_{M}$, $k=1,2$. Then

$$
\begin{aligned}
A_{1} * A_{2}= & \left(s_{1} s_{2}+t_{1} t_{2}\right)\left(\frac{s_{1} s_{2}}{s_{1} s_{2}+t_{1} t_{2}} B_{1} * B_{2}+\frac{t_{1} t_{2}}{s_{1} s_{2}+t_{1} t_{2}} C_{1} * C_{2}\right) \\
& -\left(t_{1} s_{2}+s_{1} t_{2}\right)\left(\frac{t_{1} s_{2}}{t_{1} s_{2}+s_{1} t_{2}} C_{1} * B_{2}+\frac{s_{1} t_{2}}{t_{1} s_{2}+s_{1} t_{2}} B_{1} * C_{2}\right) \\
= & \left(s_{1} s_{2}+t_{1} t_{2}\right) B^{\prime}-\left(t_{1} s_{2}+s_{1} t_{2}\right) C^{\prime} .
\end{aligned}
$$

The last expression defines $B^{\prime}$ and $C^{\prime}$; clearly both are copulas, since $\mathcal{C}$ is closed under the * product and under convex combinations. It follows that

$$
\left\|A_{1} * A_{2}\right\|_{M} \leq s_{1} s_{2}+t_{1} t_{2}+t_{1} s_{2}+s_{1} t_{2}=\left(s_{1}+t_{1}\right)\left(s_{2}+t_{2}\right)=\left\|A_{1}\right\|_{M}\left\|A_{2}\right\|_{M} .
$$

This is the desired result. 
This completes the proof of the main result concerning the Minkowski norm. Observe that continuity of the * product in each place, and also joint continuity, follow immediately from Theorem 3.2. We turn to the computation of $\|\cdot\|_{M}$ in special cases.

THEOREM 3.3. If $B$ and $C$ are any two distinct left (or right) invertible copulas, and $s$ and $t$ are any nonnegative real numbers, then $\|s B-t C\|_{M}=s+t$.

PROOF. Suppose that $B$ and $C$ are left invertible and $B \neq C$, and $s, t \geq 0$. Clearly $\|s B-t C\|_{M} \leq s+t$.

Since $B$ and $C$ are left invertible, $B_{, 1}$ and $C_{, 1}$ are 0 or 1 almost everywhere, Darsow et al. [2]. It is easy to see that the measure of the set where the first partial derivative $B_{, 1}$ is 1 is $1 / 2$. and similarly for the measure of the set where $C_{, 1}=1$. Since $B \neq C$, it follows that the sets

$$
\begin{aligned}
& F_{1}=\left\{(x, y) \mid B_{, 1}(x, y)=1 \text { and } C_{, 1}(x, y)=0\right\} \\
& F_{2}=\left\{(x, y) \mid B_{, 1}(x, y)=0 \text { and } C_{, 1}(x, y)=1\right\}
\end{aligned}
$$

have (equal) nonzero measure. Now suppose that $s^{\prime}, t^{\prime} \geq 0$ and $D, E \in \mathcal{C}$ are such that

$$
s B-t C=s^{\prime} D-t^{\prime} E .
$$

Take partial derivatives with respect to $x$ and evaluate at points $(x, y) \in F_{1}$ where the derivatives of all four copulas $B, C, D$ and $E$ exist (this is almost all points of $F_{1},[2]$ ) to obtain

$$
\left.s=s B_{, 1}(x, y)-t C_{, 1}(x, y)=s^{\prime} D_{, 1}(x, y)-t^{\prime} E_{, 1}(x, y)\right) \leq s^{\prime}
$$

using the fact that the first partial derivatives of any copula lie in $[0,1]$, wherever they exist, [2]. It follows that $s \leq s^{\prime}$. By similar argument, evaluating on $F_{2}, t \leq t^{\prime}$. Thus $s+t \leq\|s B-t C\|_{M}$. This completes the proof for left invertible copulas. The corresponding result for right invertible copulas follows by taking transposes.

It follows from the foregoing result that if $B$ and $C$ are any two distinct left (or right) invertible copulas, then $\|B-C\|_{M}=2$. The topology of the Minkowski norm, restricted to the group of invertible copulas, is accordingly the discrete topology.

The next result concerns the Minkowski norm of differences of copulas which are ordinal sums. The ordinal sum construction is as follows: Let $\left\{\left(a_{n}, b_{n}\right)\right\}_{n \in \mathcal{I}}$ be a partition of $[0,1]$, that is, a finite or countable set of disjoint open intervals the closure of whose union is the whole interval. To each $n \in \mathcal{I}$, assign a copula $A_{n}$. Define a function $A:[0,1]^{2} \rightarrow[0,1]$ via

$$
A(x, y)= \begin{cases}a_{n}+\left(b_{n}-a_{n}\right) A_{n}\left(\frac{x-a_{n}}{b_{n}-a_{n}}, \frac{y-a_{n}}{b_{n}-a_{n}}\right), & (x, y) \in\left[a_{n}, b_{n}\right]^{2} \\ M(x, y), & \text { otherwise. }\end{cases}
$$

Then $A$ is a copula, Darsow et al. [2]. $A$ is called the ordinal sum of the copulas $A_{n}$ with respect to the given partition. We will write $A=\oplus_{n \in \mathcal{I}} A_{n}$ for the ordinal sum.

THEOREM 3.4. Let $A=\oplus_{n \in \mathcal{I}} A_{n}$ and $B=\oplus_{n \in \mathcal{I}} B_{n}$ be copulas which are ordinal sums with respect to the same partition of $[0,1]$. Then

$$
\|A-B\|_{M}=\max _{n \in \mathcal{I}}\left\|A_{n}-B_{n}\right\|_{M} .
$$

PROOF. We will prove the result when $\mathcal{I}=\{1,2\}$ and $\left(a_{1}, b_{1}\right)=(0, \lambda)$ and $\left(a_{2}, b_{2}\right)=(\lambda, 1)$ for some $\lambda \in(0,1)$. The general result then follows via an inductive argument.

Thus, suppose that $A_{1}, A_{2}, B_{1}$ and $B_{2}$ are copulas and write

$$
C(x, y)=C_{1} \oplus C_{2}(x, y)= \begin{cases}\lambda C_{1}\left(\frac{x}{\lambda}, \frac{y}{\lambda}\right), & 0 \leq x, y \leq \lambda \\ \lambda+(1-\lambda) C_{2}\left(\frac{x-\lambda}{1-\lambda}, \frac{y-\lambda}{1-\lambda}\right), & \lambda \leq x, y \leq 1 \\ M(x, y), & \text { otherwise }\end{cases}
$$


where $C=A$ or $B$. Suppose $A_{1}-B_{1}=s D_{1}-t D_{2}$ where $s, t \geq 0, D_{1}, D_{2} \in \mathcal{C}$ and $\left\|A_{1}-B_{1}\right\|_{M}=$ $s+t$; evaluate at $(1,1)$ and conclude that necessarily $s=t$. Similarly, suppose $A_{2}-B_{2}=u E_{1}-v E_{2}$ where $u, v \geq 0, E_{1}, E_{2} \in \mathcal{C}$ and $\left\|A_{2}-B_{2}\right\|_{M}=u+v$; evaluate at $(1,1)$ and conclude that necessarily $u=v$. Suppose that $s>u$ (the proof is similar if $s \leq u$ ) and set

$$
F_{k}=\frac{u}{s} E_{k}+\frac{s-u}{s} M, \quad k=1,2 .
$$

Then $A_{2}-B_{2}=s F_{1}-s F_{2}$. It follows that

$$
A-B=s D_{1} \oplus F_{1}-s D_{2} \oplus F_{2}
$$

so that $\|A-B\|_{M} \leq 2 s=\max _{k=1,2}\left\|A_{k}-B_{k}\right\|_{M}$.

The opposite inequality utilizes a construction we have occasionally found useful in trying to work with the Minkowski norm. Suppose that $A-B=s D-t E$, where $s, t \geq 0, D, E \in \mathcal{C}$ and $\|A-B\|_{M}=s+t$; evaluate at $(1,1)$ and conclude that $s=t$; evaluate at $(x, \lambda)$ and $(\lambda, y)$ and conclude (using the fact that, by (3.1), $A$ and $B$ agree at $(x, \lambda)$ and $(\lambda, y)$ )

$$
\begin{array}{ll}
D(x, \lambda)=E(x, \lambda) & \text { for all } x \\
D(\lambda, y)=E(\lambda, y) & \text { for all } y
\end{array}
$$

We want to show that $\left\|A_{k}-B_{k}\right\|_{M} \leq 2 s, k=1,2$. We will show that the result holds for $k=1$; the proof for $k=2$ is similar.

Observe first that

$$
\begin{aligned}
& \frac{\partial}{\partial x}\left(\frac{1}{\lambda} D(\lambda x, \lambda y)\right)=D_{, 1}(\lambda x, \lambda y) \leq 1 \\
& \frac{\partial}{\partial y}\left(\frac{1}{\lambda} D(\lambda x, \lambda y)\right)=D_{, 2}(\lambda x, \lambda y) \leq 1 .
\end{aligned}
$$

It follows that if for any $\epsilon>0$ we set

$$
\begin{aligned}
& \phi(x)=\left((1+\epsilon) x-\frac{1}{\lambda} D(\lambda x, \lambda)\right) / \sqrt{1+\epsilon-\frac{1}{\lambda} D(\lambda, \lambda)} \\
& \psi(y)=\left((1+\epsilon) y-\frac{1}{\lambda} D(\lambda, \lambda y)\right) / \sqrt{1+\epsilon-\frac{1}{\lambda} D(\lambda, \lambda)}
\end{aligned}
$$

the functions $\phi$ and $\psi$ are strictly increasing on $[0,1]$. (Observe that necessarily $D(\lambda, \lambda) \leq \lambda$; this is a basic property of copulas, Darsow et al. [2].) To complete the construction, define for all $(x, y) \in[0,1]^{2}$

$$
\begin{aligned}
& \tilde{D}_{1}(x, y)=\phi(x) \psi(y)+\frac{1}{\lambda} D(\lambda x, \lambda y) \\
& \tilde{E}_{1}(x, y)=\phi(x) \psi(y)+\frac{1}{\lambda} E(\lambda x, \lambda y) .
\end{aligned}
$$

It is readily verified that $D_{1}=\tilde{D}_{1} /(1+\epsilon)$ and $E_{1}=\tilde{E}_{1} /(1+\epsilon)$ are copulas; monotonocity follows from the fact that $D$ and $E$ are copulas and the fact that $\phi$ and $\psi$ are increasing; the boundary conditions for $D_{1}$ are verified by direct calculation, and those for $E_{1}$ follow from (3.2) and (3.3).

The desired result now follows easily. For all $x, y \in[0,1]$ we have by construction

$$
\begin{aligned}
A_{1}(x, y)-B_{1}(x, y) & =\frac{1}{\lambda} A(\lambda x, \lambda y)-\frac{1}{\lambda} B(\lambda x, \lambda y) \quad \text { (using (3.1)) } \\
& =\frac{s}{\lambda} D(\lambda x, \lambda y)-\frac{s}{\lambda} E(\lambda x, \lambda y) \\
& =(1+\epsilon) s D_{1}(x, y)-(1+\epsilon) s E_{1}(x, y) .
\end{aligned}
$$


Thus, for any $\epsilon>0,\left\|A_{1}-B_{1}\right\|_{M} \leq(1+\epsilon) 2 s=(1+\epsilon)\|A-B\|_{M}$. The theorem follows. $\mid$

Since span $(\mathcal{C})$ is a Banach algebra under $\|\cdot\|_{M}$, the Minkowski norm is a natural one, and the issue of how to compute it becomes important. We will return to the issue of how to compute the Minkowski norm again in Theorem 5.2.

\section{SOBOLEV NORMS.}

We will work with the Sobolev seminorm $|A|_{1, p}$ given by

$$
|A|_{1, p}= \begin{cases}\left(\int_{0}^{1} \int_{0}^{1}\left(\left|A_{, 1}(x, y)\right|^{p}+\left|A_{, 2}(x, y)\right|^{p}\right) d x d y\right)^{1 / p}, & p \in[1, \infty) \\ \max \left\{\operatorname{ess} \sup \left|A_{, 1}(x, y)\right|, \text { ess } \sup \left|A_{, 2}(x, y)\right|\right\}, & p=\infty .\end{cases}
$$

THEOREM 4.1. For all $p \in[1, \infty],|\cdot|_{1, p}$ is a norm on span $(\mathcal{C})$ equivalent to $\|\cdot\|_{1, p}$.

PROOF. This follows from the fact that an element $A \in \operatorname{span}(\mathcal{C})$ necessarily vanishes on the lower and left boundaries of $[0,1]^{2}$. The details of the proof can be found in Adams [1].

One of our principal results is that the * product restricted to $\mathcal{C}$ is continuous in each place and jointly continuous in the topology of $|\cdot|_{1, p}$, for $p \in[1, \infty)$. Whether or not the corresponding result holds for $p=\infty$ is an open issue, and what can be said about continuity of the * product on all of span $(\mathcal{C})$ is also an open issue. Another principal result is that $\mathcal{C}$ is a closed subset of $W^{1, p}$ for all $p \in[1, \infty]$, so that $\mathcal{C}$ is a complete metric space with respect to each of the Sobolev norms considered. Putting the principal results together, we have that $\mathcal{C}$ is a complete metric space under $|\cdot|_{1, p}$, for $p \in[1, \infty)$ and that the product behaves well with respect to the metric.

The utility of the Sobolev norms on span $(\mathcal{C})$ is much less clear, both because the behavior of the $*$ product is not known, as noted above, and because, as we shall show, span $(\mathcal{C})$ is not complete with respect to any of the Sobolev norms. This latter result will be proved by way of a result of independent interest - the Sobolev norms on span $(\mathcal{C})$ are dominated by, but not equivalent to, the Minkowski norm.

LEMMA 4.1. The topologies induced on $\mathcal{C}$ by the norms $|\cdot|_{1, p}$ coincide for all $p \in[1, \infty)$.

PROOF. We will show that for any $p>1$, the topology induced by $|\cdot|_{1, p}$ coincides with the topology induced by $|\cdot|_{1,1}$. Since the first partial derivatives of a copula lie between 0 and 1 almost surely, Darsow et al. [2], we have $|B-C|_{1, p} \leq|B-C|_{1,1}^{1 / p}$ for any copulas $B$ and $C$. Also, by by Hölder's inequality, $|B-C|_{1,1} \leq|B-C|_{1, p}$. It follows that a ball in $\mathcal{C}$ in the topology of $|\cdot|_{1,1}$ necessarily contains a ball in the topology of $|\cdot|_{1, p}$ and vice versa.

The topology of $|\cdot|_{1, \infty}$ restricted to $\mathcal{C}$ is different from the topology of $|\cdot|_{1,1}$. To see this, let $B_{n}$ denote the ordinal sum

$$
B_{n}(x, y)= \begin{cases}n x y, & 0 \leq x, y \leq 1 / n \\ \min \{x, y\}, & \text { otherwise }\end{cases}
$$

Then by direct calculation

$$
\begin{aligned}
& \left|B_{n}-M\right|_{1,1}=\frac{2}{3 n^{2}} \rightarrow 0 \text { whereas } \\
& \left|B_{n}-M\right|_{1, \infty}=1 \text { for all } n .
\end{aligned}
$$

Roughly, the topology of $|\cdot|_{1, p}$, for $p \in[1, \infty)$, is the topology of pointwise convergence of derivatives, since pointwise convergence of derivatives implies convergence in the sense of $|\cdot|_{1, p}$ for $p \in[1, \infty)$, by the dominated convergence theorem, and convergence in the sense of $|\cdot|_{1, p}$ implies the existence of a subsequence whose first partial derivatives converge pointwise a.e. The topology of $|\cdot|_{1, \infty}$, on the other hand, is the topology of uniform convergence of derivatives.

For $B \in \mathcal{C}$ and $f \in L^{1}([0,1])$ define

$$
B \hat{*} f(x)=\int_{0}^{1} B, 2(x, t) f(t) d t
$$


It is easy to verify that for $f \in L^{\infty}, B \hat{*} f$ is Lipschitz continuous with Lipschitz constant $\|f\|_{0, \infty}$ and thus is absolutely continuous.

LEMMA 4.2. For all $B \in \mathcal{C}$ and $f \in L^{1}([0,1]),\left|\frac{d}{d x} B \hat{*} f\right| \leq \frac{d}{d x} B \hat{*}|f|$ a.e.

PROOF. Since $x \rightarrow B_{, 2}(x, t)$ is almost surely nondecreasing, Darsow et al. [2], $x \rightarrow B \hat{*} f$ is nondecreasing whenever $f$ is nonnegative. The desired result follows immediately from the fact that for any $f \in L^{1},|f|-f$ and $|f|+f$ are both nonnegative.

THEOREM 4.2. The * product on $\mathcal{C}$ is continuous in each place and also jointly continuous in the topology of $|\cdot|_{1, p}$ for all $p \in[1, \infty)$.

PROOF. By Lemma 4.1, it suffices to consider the case $p=1$. Let $B_{n}, B$ and $C$ be copulas, and suppose $\left|B-B_{n}\right|_{1,1} \rightarrow 0$. We show first that $\left|C * B-C * B_{n}\right|_{1,1} \rightarrow 0$ and $\left|B * C-B_{n} * C\right|_{1,1} \rightarrow 0$. This will prove that $*$ is continuous in each place.

We will show that $\left\|(B * C)_{, 1}-\left(B_{n} * C\right)_{, 1}\right\|_{0,1} \rightarrow 0$ and $\left\|(C * B)_{, 1}-\left(C * B_{n}\right)_{, 1}\right\|_{0,1} \rightarrow 0$. The proof for partial derivatives with respect to the second argument is similar.

Given $\epsilon>0$, let $\phi \in C_{0}^{\infty}\left([0,1]^{2}\right)$ be such that $\left\|C_{, 1}-\phi\right\|_{0,1}<\epsilon / 3 . C_{0}^{\infty}\left([0,1]^{2}\right)$ is the space of $C^{\infty}$ functions on the unit square which vanish on the boundary. We can find such a $\phi$ since $C_{0}^{\infty}$ is dense in $L^{1}$, e.g. Adams [1]. Then

$$
\begin{aligned}
\left\|(B * C)_{, 1}-\left(B_{n} * C\right)_{, 1}\right\|_{0,1}= & \int_{0}^{1} \int_{0}^{1}\left|\frac{d}{d x} \int_{0}^{1}\left(B-B_{n}\right)_{, 2}(x, s) C_{, 1}(s, t) d s\right| d t d x \\
\leq & \int_{0}^{1} \int_{0}^{1}\left|\frac{d}{d x} \int_{0}^{1}\left(B-B_{n}\right)_{, 2}(x, s) \phi(s, t) d s\right| d t d x \\
& +\int_{0}^{1} \int_{0}^{1}\left|\frac{d}{d x} \int_{0}^{1} B_{, 2}(x, s)\left(\phi-C_{, 1}\right)(s, t) d s\right| d t d x \\
& +\int_{0}^{1} \int_{0}^{1}\left|\frac{d}{d x} \int_{0}^{1} B_{n, 2}(x, s)\left(\phi-C_{, 1}\right)(s, t) d s\right| d t d x \\
\leq & \int_{0}^{1} \int_{0}^{1}\left|\frac{d}{d x} \int_{0}^{1}\left(B-B_{n}\right)(x, s) \phi_{, 1}(s, t) d s\right| d t d x \\
& +\int_{0}^{1} \int_{0}^{1} \frac{d}{d x} \int_{0}^{1} B_{, 2}(x, s)\left|\left(\phi-C_{, 1}\right)(s, t)\right| d s d t d x \\
& +\int_{0}^{1} \int_{0}^{1} \frac{d}{d x} \int_{0}^{1} B_{n, 2}(x, s)\left|\left(\phi-C_{, 1}\right)(s, t)\right| d s d t d x .
\end{aligned}
$$

Here, we integrated by parts in the first term in the second expression on the right, and we used Lemma 4.2 to estimate the last two terms in the expression. Since $\phi \in C^{\infty}$, we can perform the integration with respect to $x$ in the last two terms to obtain the estimate

$$
\begin{aligned}
\left\|(B * C)_{, 1}-\left(B_{n} * C\right)_{, 1}\right\|_{0,1} \leq & \left\|\phi_{, 1}\right\|_{0, \infty} \int_{0}^{1} \int_{0}^{1}\left|\left(B-B_{n}\right)_{, 1}(x, s)\right| d s d x \\
& +2 \int_{0}^{1} \int_{0}^{1}\left|\left(\phi-C_{, 1}\right)(s, t)\right| d s d t \\
\leq & \left\|\phi_{, 1}\right\|_{0, \infty}\left|B-B_{n}\right|_{1,1}+2 \epsilon / 3 .
\end{aligned}
$$

Choose $N$ so large that $n \geq N$ implies

$$
\left\|\phi_{, 1}\right\|_{0, \infty}\left|B-B_{n}\right|_{1,1}<\epsilon / 3 .
$$

Then for all such $n$,

$$
\left\|(B * C)_{, 1}-\left(B_{n} * C\right)_{, 1}\right\|_{0,1}<\epsilon
$$

so that $\left\|(B * C)_{, 1}-\left(B_{n} * C\right)_{, 1}\right\|_{0,1} \rightarrow 0$. 
To complete the proof, observe that

$$
\begin{aligned}
\left\|(C * B)_{, 1}-\left(C * B_{n}\right)_{, 1}\right\|_{0,1} & =\int_{0}^{1} \int_{0}^{1}\left|\frac{d}{d x} \int_{0}^{1} C_{, 2}(x, s)\left(B-B_{n}\right)_{, 1}(s, t) d s\right| d t d x \\
& \leq \int_{0}^{1} \int_{0}^{1} \frac{d}{d x} \int_{0}^{1} C_{, 2}(x, s)\left|\left(B-B_{n}\right)_{, 1}(s, t)\right| d s d t d x \\
& =\int_{0}^{1} \int_{0}^{1}\left|\left(B-B_{n}\right)_{, 1}(s, t)\right| d s d t \\
& \leq\left|B-B_{n}\right|_{1,1}
\end{aligned}
$$

Here, we used Lemma 4.2 and performed the integration with respect to $x$. It follows that $\left\|(C * B)_{, 1}-\left(C * B_{n}\right)_{, 1}\right\|_{0,1} \rightarrow 0$.

Thus, $*$ is continuous in each place with respect to the topology of $|\cdot|_{1, p}$ for $p \in[1, \infty)$.

Observe that the argument just given shows that for any three copulas $B, C$ and $D$,

$$
\left\|(C * B)_{, 1}-(C * D)_{, 1}\right\|_{0,1} \leq|B-D|_{1,1} .
$$

We will make use of this observation to show joint continuity. To that end, let $B_{n}, B, C_{n}$ and $C$ be copulas, and suppose that $\left|B-B_{n}\right|_{1,1} \rightarrow 0$ and $\left|C-C_{n}\right|_{1,1} \rightarrow 0$. We want to show that $\left|B * C-B_{n} * C_{n}\right|_{1,1} \rightarrow 0$. Write $B * C-B_{n} * C_{n}=B_{n} *\left(C-C_{n}\right)+\left(B-B_{n}\right) * C$. Then

$$
\begin{aligned}
\left\|(B * C)_{, 1}-\left(B_{n} * C_{n}\right)_{, 1}\right\|_{0,1} & \leq\left\|\left[B_{n} *\left(C-C_{n}\right)\right]_{, 1}\right\|_{0,1}+\left\|\left[\left(B-B_{n}\right) * C\right]_{, 1}\right\|_{0,1} \\
& \leq\left|C-C_{n}\right|_{1,1}+\left\|\left[\left(B-B_{n}\right) * C\right]_{, 1}\right\|_{0,1} .
\end{aligned}
$$

We have used (4.3) here. The first term on the right goes to zero by hypothesis and the second term goes to zero by the onesided continuity of the $*$ operation proved above. Thus, $\|(B * C)_{, 1}-$ $\left(B_{n} * C_{n}\right)_{, 1} \|_{0,1} \rightarrow 0$. By an analogous argument, $\left\|(B * C)_{, 2}-\left(B_{n} * C_{n}\right)_{, 2}\right\|_{0,1} \rightarrow 0$. Thus, $\left|B * C-B_{n} * C_{n}\right|_{1,1} \rightarrow 0$, and joint continuity is established.

We turn now to completeness issues.

THEOREM 4.3. For all $p \in[1, \infty]$ the topology of $|\cdot|_{1, p}$ restricted to $\mathcal{C}$ is stronger than the topology of uniform convergence.

PROOF. For $p>2,\|\cdot\|_{1, p}$, and thus $|\cdot|_{1, p}$, dominates the uniform norm $\|\cdot\|_{0, \infty}$, by the Sobolev embedding theorem, Adams [1]. Thus, for each $p>2$, there is a constant $C_{p}$ such that

$$
\|A\|_{0, \infty} \leq C_{p}|A|_{1, p}
$$

for all $A \in \operatorname{span}(\mathcal{C})$. This implies the desired result, for $p>2$. Since by Lemma 4.1 the topologies of $|\cdot|_{1, p}$ restricted to $\mathcal{C}$ coincide for $p \in[1, \infty)$, the conclusion of the theorem holds also for $p \in[1,2]$, though an inequality of the form (4.4) need not hold for $p \in[1,2]$.

Observe that the topologies of $|\cdot|_{1, p}$ are strictly stronger than that of $\|\cdot\|_{0, \infty}$, since if the topologies coincided, the $*$ product would be jointly continuous in the latter topology. This is not the case, however.

There is a sharper statement which implies Theorem 4.3 and which we will make use of later:

THEOREM 4.4. For all $p \in(1, \infty)$ the weak topology of $W^{1, p}$ restricted to $\mathcal{C}$ coincides with the topology of uniform convergence.

PROOF. A sequence of copulas is weakly Cauchy in the sense of $W^{1, p}$ if and only if it is uniformly Cauchy, and the weak and uniform limits are the same. This follows easily from the fact that $\mathcal{C}$ is a compact subset of $L^{\infty}$ and from the fact that the unit ball in $L^{p}, p \in(1, \infty)$ (which contains both first partial derivatives of any copula) is weakly sequentially compact. I

THEOREM 4.5. For all $p \in[1, \infty], \mathcal{C}$ is a complete metric space under the metric

$$
d(B, C)=|B-C|_{1, p}
$$


PROOF. This is a corollary of Theorem 4.3. Suppose $B$ is in the closure of $\mathcal{C}$ in $W^{1 . p}$, and let $B_{n} \in \mathcal{C}$ be such that $\left|B-B_{n}\right|_{1, p} \rightarrow 0$. We want to show $B \in \mathcal{C}$. By Theorem $4.3\left\|B-B_{n}\right\|_{0, \infty} \rightarrow 0$ and the uniform limit of a sequence of copulas is a copula, so $B \in \mathcal{C}$.

We turn now to consideration of the Sobolev norms on all of span $(\mathcal{C})$.

THEOREM 4.6. For $p \in[1, \infty]$, the Minkowski norm dominates the norm $|\cdot|_{1, p}$ on $\operatorname{span}(\mathcal{C})$.

PROOF. Since for $p \in[1, \infty),|A|_{1, p} \leq 2^{1 / p}|A|_{1, \infty}$, it suffices to establish the result for $p=\infty$. Since the first partial derivatives of a copula lie in $[0,1]$ a.s., the result for $p=\infty$ follows via an argument similar to that of Lemma 3.1. I

To investigate the issue of whether span $(\mathcal{C})$ is complete under any of the Sobolev norms, we use the following easy consequence of the open mapping theorem (Rudin [5]):

LEMMA 4.3. Let $\mathcal{B}$ be a Banach space under each of two norms. If one of the norms dominates the other, the two norms are equivalent.

It follows from Theorem 4.6 and Lemma 4.3 that $\operatorname{span}(\mathcal{C})$ is complete under one of the Sobolev norms if and only if that norm is equivalent to $\|\cdot\|_{M}$. A counterexample given in Example 5.1 below shows that the norms are not equivalent. By Theorem 4.6 and Lemma 4.3, therefore, span $(\mathcal{C})$ is not complete under any of the Sobolev norms. We can always work with the closure of $\operatorname{span}(\mathcal{C})$ in $W^{1, p}$, if we wish, but the subspace span $(\mathcal{C})$ itself is not closed.

Before leaving the Sobolev norms, we comment on the difficulty of extending Theorem 4.2, concerning continuity of the $*$ product, to all of span $(\mathcal{C})$. Since as noted above the norms $\|\cdot\|_{M}$ and $|\cdot|_{1, p}$ are not equivalent, there exist sequences $A_{n}=s_{n} B_{n}-t_{n} C_{n}$ which are Cauchy with respect to $|\cdot|_{1, p}$ for which $s_{n}+t_{n}$ is an unbounded sequence. This cannot be accomodated within the framework of the proof we have given of Theorem 4.2. If, therefore, the $*$ product is continuous on all of span $(\mathcal{C})$ in the topology of $|\cdot|_{1, p}$, it will have to be shown by some modified or completely different line of proof.

\section{JORDAN NORM}

We can say less about the Jordan norm than about the norms considered above. The question of how the * product behaves with respect to the topology of the Jordan norm is open. The principal interest of the norm is that it sheds light on the behavior of the other norms.

THEOREM 5.1. The Jordan norm dominates the uniform norm on span $(\mathcal{C})$ and is dominated by the Minkowski norm.

PROOF. Let $A \in \operatorname{span}(\mathcal{C})$. Write $\mu_{A}$ for the finite signed measure induced by $A$ on $[0,1]^{2}$, write $\mu_{A}=\mu_{A}^{+}-\mu_{A}^{-}$for its Jordan decomposition, where $\mu_{A}^{ \pm}(F)=\mu_{A}\left(F \cap E^{ \pm}\right)$, and write $\left|\mu_{A}\right|=\mu_{A}^{+}+\mu_{A}^{-}$. Then

$$
\|A\|_{J}=\left|\mu_{A}\right|\left([0,1]^{2}\right) .
$$

Observe that for any $(x, y) \in[0,1]^{2}$ we have

$$
|A(x, y)|=\left|\mu_{A}([0, x] \times[0, y])\right| \leq\left|\mu_{A}\right|([0, x] \times[0, y]) \leq\|A\|_{J} .
$$

Take the supremum over $(x, y) \in[0,1]^{2}$ and conclude that $\|A\|_{0, \infty} \leq\|A\|_{J}$.

Next, observe that if $A=s B-t C$, where $s, t \geq 0, B, C \in \mathcal{C}$ and $\|A\|_{M}=s+t$, we have

$$
\begin{aligned}
& \mu_{A}^{+}\left([0,1]^{2}\right)=\mu_{A}\left(E^{+}\right)=s \mu_{B}\left(E^{+}\right)-t \mu_{C}\left(E^{+}\right) \leq s \mu_{B}\left(E^{+}\right) \leq s \\
& \mu_{A}^{-}\left([0,1]^{2}\right)=-\mu_{A}\left(E^{-}\right)=-s \mu_{B}\left(E^{-}\right)+t \mu_{C}\left(E^{-}\right) \leq t \mu_{B}\left(E^{-}\right) \leq t
\end{aligned}
$$

using the fact that $\mu_{B}$ and $\mu_{C}$ are probability measures and that $\mu_{A}=s \mu_{B}-t \mu_{C}$. Sum the foregoing inequalities and conclude that $\|A\|_{J} \leq s+t=\|A\|_{M}$.

We have a representation of the Minkowski norm which makes use of the Jordan decomposition: 
THEOREM 5.2. Let $A \in \operatorname{span}(\mathcal{C})$, let $\mu_{A}=\mu_{A}^{+}-\mu_{A}^{-}$be the Jordan decomposition of the finite signed measure induced by $A$, as above, and set $A^{+}(x, y)=\mu_{A}^{+}([0, x] \times[0, y])$, and $A^{-}(x, y)=\mu_{A}^{-}([0, x] \times[0, y])$. Then

$$
\|A\|_{M}=\max \left\{\left\|A_{, 1}^{+}(\cdot, 1)\right\|_{\infty},\left\|A_{, 2}^{+}(1, \cdot)\right\|_{\infty}\right\}+\max \left\{\left\|A_{, 1}^{-}(\cdot, 1)\right\|_{\infty},\left\|A_{, 2}^{-}(1, \cdot)\right\|_{\infty}\right\} .
$$

PROOF. $A^{+}$and $A^{-}$are continuous monotonic functions satisfying

$$
A^{+}(x, 0)=A^{+}(0, y)=A^{-}(x, 0)=A^{-}(0, y)=0 .
$$

These facts are easy to verify. They differ from copulas in that they satisfy different boundary conditions on the upper and right boundaries of the unit square.

If $A=s B-t C$ where $B, C \in \mathcal{C}$ and $s, t \geq 0$, and $\|A\|_{M}=s+t$, then for $x_{1}<x_{2}$

$$
\begin{aligned}
A^{+}\left(x_{2}, y\right)-A^{+}\left(x_{1}, y\right) & =s \mu_{B}\left(E^{+} \cap\left(x_{1}, x_{2}\right] \times[0, y]\right)-t \mu_{C}\left(E^{+} \cap\left(x_{1}, x_{2}\right] \times[0, y]\right) \\
& \leq s \mu_{B}\left(E^{+} \cap\left(x_{1}, x_{2}\right] \times[0, y]\right) \\
& \leq s \mu_{B}\left(\left(x_{1}, x_{2}\right] \times[0, y]\right) \\
& \leq s\left(B\left(x_{2}, y\right)-B\left(x_{1}, y\right)\right) \\
& \leq s\left(x_{2}-x_{1}\right)
\end{aligned}
$$

using the Lipschitz condition satisfied by the copula $B$. Since sections of $A^{+}$are nondecreasing, first partial derivatives exist almost everywhere, and the foregoing inequalities imply

$$
0 \leq A_{, 1}^{+}(x, y) \leq s \text { a.e. }
$$

By similar reasoning,

$$
\begin{array}{ll}
0 \leq A_{, 2}^{+}(x, y) \leq s & \text { a.e. } \\
0 \leq A_{, 1}^{-}(x, y) \leq t & \text { a.e. } \\
0 \leq A_{, 2}^{-}(x, y) \leq t & \text { a.e. }
\end{array}
$$

We use the notation "a.e." in the statements above as shorthand for the more precise statements that, for all $y,(5.2 \mathrm{a})$ and (5.3a) hold for almost all $x$ and that, for all $x,(5.2 \mathrm{~b})$ and (5.3b) hold for almost all $y$. It follows from (5.2) and (5.3) that $\|A\|_{M}=s+t$ dominates the right hand side of (5.1), which in particular must be finite.

To obtain the converse inequality, we make use again of the construction utilized in the proof of Theorem 3.4. Observe that, since $A^{-}=A^{+}-A$ and $A$ is linear on the upper and right boundaries of $[0,1]^{2}$, we can write

$$
\begin{aligned}
& \frac{1}{x_{2}-x_{1}}\left(A^{-}\left(x_{2}, 1\right)-A^{-}\left(x_{1}, 1\right)\right)=\frac{1}{x_{2}-x_{1}}\left(A^{+}\left(x_{2}, 1\right)-A^{+}\left(x_{1}, 1\right)\right)-A(1,1) \\
& \frac{1}{y_{2}-y_{1}}\left(A^{-}\left(1, y_{2}\right)-A^{-}\left(1, y_{1}\right)\right)=\frac{1}{y_{2}-y_{1}}\left(A^{+}\left(1, y_{2}\right)-A^{+}\left(1, y_{1}\right)\right)-A(1,1)
\end{aligned}
$$

so that also

$$
\begin{aligned}
& \max \left\{\operatorname{ess} \sup \left\{A_{, 1}^{-}(x, 1)\right\}, \text { ess } \sup \left\{A_{, 2}^{-}(1, y)\right\}\right\} \\
& \quad=\max \left\{\text { ess } \sup \left\{A_{, 1}^{+}(x, 1)\right\}, \text { ess } \sup \left\{A_{, 2}^{+}(1, y)\right\}\right\}-A(1,1)
\end{aligned}
$$

The right hand side of this expression is necessarily nonnegative. Let

$$
\kappa=\max \left\{\operatorname{ess} \sup \left\{A_{, 1}^{+}(x, 1)\right\}, \operatorname{ess} \sup _{, 2}\left\{A_{, 2}^{+}(1, y)\right\}\right\} .
$$


For any $\epsilon>0$, set

$$
\begin{aligned}
& \phi(x)=\left((\kappa+\epsilon) x-A^{+}(x, 1)\right) /\left(\kappa+\epsilon-A^{+}(1,1)\right)^{1 / 2} \\
& \psi(y)=\left((\kappa+\epsilon) y-A^{+}(1, y)\right) /\left(\kappa+\epsilon-A^{+}(1,1)\right)^{1 / 2} \\
& B(x, y)=\frac{1}{\kappa+\epsilon}\left(A^{+}(x, y)+\phi(x) \psi(y)\right) \\
& C(x, y)=\frac{1}{\kappa+\epsilon-A(1,1)}\left(A^{-}(x, y)+\phi(x) \psi(y)\right) .
\end{aligned}
$$

Then $\phi$ and $\psi$ are strictly increasing functions, and, by reasoning similar to that used in the proof of Theorem 3.4, both $B$ and $C$ are copulas. Since by construction

$$
A=(\kappa+\epsilon) B-(\kappa+\epsilon-A(1,1)) C
$$

it follows that

$$
\|A\|_{M} \leq 2 \kappa+2 \epsilon-A(1,1) .
$$

Since $\epsilon>0$ is arbitrarily small, we obtain finally

$$
\begin{aligned}
\|A\|_{M} & \leq 2 \kappa-A(1,1) \\
& \leq \max \left\{\left\|A_{, 1}^{+}(\cdot, 1)\right\|_{\infty},\left\|A_{, 2}^{+}(1, \cdot)\right\|_{\infty}\right\}+\max \left\{\left\|A_{, 1}^{-}(\cdot, 1)\right\|_{\infty},\left\|A_{, 2}^{-}(1, \cdot)\right\|_{\infty}\right\}
\end{aligned}
$$

using (5.4) and the definition of $\kappa$. Thus, the right hand side of (5.1) dominates $\|A\|_{M}$. I

We now give two examples. In the first, we construct a sequence of elements of $\operatorname{span}(\mathcal{C})$ on which the norms $\|\cdot\|_{J}$ and $\|\cdot\|_{M}$ are the same, and both diverge to $+\infty$, whereas the norm $|\cdot|_{1, \infty}$ is bounded. In the second, we construct a sequence of elements of span $(\mathcal{C})$ for which the norms $\|\cdot\|_{J}$ and $\|\cdot\|_{0, \infty}$ are essentially the same, and both converge to zero, whereas the norms $|\cdot|_{1, \infty}$ and $\|\cdot\|_{M}$ diverge to $+\infty$. We shall then comment on the examples.

EXAMPLE 5.1. Let $n$ be an even integer. Partition $[0,1]^{2}$ into $n^{2}$ disjoint squares of side $1 / n$ : let $x_{k}=y_{k}=k / n, k=0, \ldots, n$ and write

$$
R_{\jmath k}= \begin{cases}{\left[x_{\jmath-1}, x_{\jmath}\right) \times\left[y_{k-1}, y_{k}\right),} & 1 \leq j, k<n \\ {\left[x_{\jmath-1}, x_{\jmath}\right] \times\left[y_{k-1}, y_{k}\right),} & j=n, 1 \leq k<n \\ {\left[x_{\jmath-1}, x_{\jmath}\right) \times\left[y_{k-1}, y_{k}\right],} & k=n, 1 \leq j<n \\ {\left[x_{\jmath-1}, x_{j}\right] \times\left[y_{k-1}, y_{k}\right],} & j=k=n\end{cases}
$$

Define a finite signed measure $\mu_{n}$ on $[0,1]^{2}$ via the conditions that $\mu_{n}\left(R_{j k}\right)=(-1)^{j+k} / n$ and that the mass of $\mu_{n}$ be spread uniformly on each $R_{j k}$. That is, define $\mu_{n}$ via

$$
\mu_{n}\left(F \cap R_{j k}\right)=\frac{(-1)^{j+k} \lambda\left(F \cap R_{j k}\right)}{n \lambda\left(R_{j k}\right)}=n(-1)^{j+k} \lambda\left(F \cap R_{j k}\right)
$$

for any measurable set $F$, where $\lambda$ denotes Lebesgue measure on $[0,1]^{2}$.

The Jordan decomposition of $\mu_{n}$ is as follows: Set

$$
\begin{aligned}
& E^{+}=\cup_{j+k \text { even }} R_{j k} \\
& E^{-}=\cup_{j+k \text { odd }} R_{j k} .
\end{aligned}
$$

Then $\mu_{n}^{+}(F)=\mu_{n}\left(F \cap E^{+}\right)$and $\mu_{n}^{-}(F)=-\mu_{n}\left(F \cap E^{-}\right)$.

Observe that

$$
\begin{aligned}
& \mu_{n}^{+}([0, x] \times[0,1])=\frac{n x}{2} \\
& \mu_{n}^{+}([0,1] \times[0, y])=\frac{n y}{2}
\end{aligned}
$$


so that $B_{n}^{+}$defined by

$$
B_{n}^{+}(x, y)=\frac{2}{n} \mu_{n}^{+}([0, x] \times[0, y])
$$

is a copula. Equations (5.6) and (5.7) are satisfied with $\mu_{n}^{-}$in place of $\mu_{n}^{+}$, so that

$$
B_{n}^{-}(x, y)=\frac{2}{n} \mu_{n}^{-}([0, x] \times[0, y])
$$

is a copula. If we define $A_{n}$ via

$$
A_{n}(x, y)=\mu_{n}([0, x] \times[0, y])
$$

we have

$$
A_{n}=\frac{n}{2} B_{n}^{+}-\frac{n}{2} B_{n}^{-} \in \operatorname{span}(\mathcal{C}) .
$$

It is easy to see that $\left|\mu_{n}\right|=n \lambda$, so that $\left\|A_{n}\right\|_{J}=n$. Using formula (5.1), together with (5.5) and (5.6) and the analogous formulae for $\mu_{n}^{-}$, we obtain also $\left\|A_{n}\right\|_{M}=n$.

We wish to compute $\left|A_{n}\right|_{1, \infty}$. For $(x, y) \in R_{\jmath k}$ we calculate, using (5.5)

$$
\begin{aligned}
A_{n}(x, y)=\frac{1}{n} & \sum_{\ell=1}^{j-1} \sum_{m=1}^{k-1}(-1)^{\ell+m}+\left(x-x_{j-1}\right) \sum_{m=1}^{k-1}(-1)^{j+m} \\
& +\left(y-y_{k-1}\right) \sum_{\ell=1}^{j-1}(-1)^{\ell+\jmath}+n\left(x-x_{j-1}\right)\left(y-y_{k-1}\right)(-1)^{\jmath+k}
\end{aligned}
$$

It follows that for $(x, y)$ in the interior of $R_{\jmath k}$ we have

$$
\left|A_{n, 1}(x, y)\right|=\left|\sum_{m=1}^{k-1}(-1)^{j+m}+n\left(y-y_{k-1}\right)(-1)^{\jmath+k}\right| \leq 2
$$

since $\left|y-y_{k-1}\right|<1 / n$ and

$$
\sum_{m=1}^{k-1}(-1)^{j+m}=-1,0 \text { or } 1 .
$$

Similarly, $\left|A_{n, 2}(x, y)\right| \leq 2$ for all $(x, y)$ interior to one of the $R_{j k}$ 's. It follows that $\left|A_{n}\right|_{1, \infty} \leq 2$.

Thus, as $n$ varies over the even positive integers, we obtain a sequence of elements in span $(\mathcal{C})$ whose Minkowski and Jordan norms are equal, and diverge to $+\infty$, but whose $W^{1, \infty}$ norms are uniformly bounded above by 2 .

EXAMPLE 5.2. Let $B_{n}$ be the ordinal sum of (4.2) above, and define $A_{n}=s_{n} M-t_{n} B_{n}$ where $s_{n}=t_{n}=n^{\alpha}$, for some positive number $\alpha$. Thus,

$$
A_{n}(x, y)= \begin{cases}0, & x \geq 1 / n \text { or } y \geq 1 / n \\ n^{\alpha}(x-n x y), & 0 \leq x \leq y \leq 1 / n \\ n^{\alpha}(y-n x y), & 0 \leq y \leq x \leq 1 / n\end{cases}
$$

Observe that $A_{n} \in \operatorname{span}(\mathcal{C})$ for all $n$. We calculate $\left\|A_{n}\right\|_{0, \infty},\left|A_{n}\right|_{1, \infty},\left\|A_{n}\right\|_{J}$ and $\left\|A_{n}\right\|_{M}$.

An easy calculation shows that

$$
\left|A_{n}\right|_{1, \infty}=n^{\alpha} .
$$

It is easy to verify that the maximum value of $\left|A_{n}(x, y)\right|$ occurs at $(x, y)=(1 / 2 n, 1 / 2 n)$, so that

$$
\left\|A_{n}\right\|_{0, \infty}=n^{\alpha-1} / 4 .
$$

The other norms are also not too difficult to calculate. Write $\lambda_{1}$ for Lebesgue measure on the line segment $L=\{(x, x) \mid 0 \leq x \leq 1\}$, normalized so that $\lambda_{1}(L)=1$; write $L_{n}$ for the line segment 
$L_{n}=\{(x, x) \mid 0 \leq x \leq 1 / n\}$; and write $\lambda_{2}$ for Lebesgue measure on $[0,1]^{2}$. Then it is easy to verify that the Jordan decomposition of $\mu_{n}=\mu_{A_{n}}$ is given by

$$
\begin{aligned}
& \mu_{n}^{+}(F)=n^{\alpha} \lambda_{1}\left(F \cap L_{n}\right) \\
& \mu_{n}^{-}(F)=n^{\alpha+1} \lambda_{2}\left(F \cap[0,1 / n]^{2}\right) .
\end{aligned}
$$

It follows that

$$
\left\|A_{n}\right\|_{J}=\left|\mu_{n}\right|\left([0,1]^{2}\right)=2 n^{\alpha-1}
$$

It follows also that

$$
\begin{aligned}
& \mu_{n}^{+}([0, x] \times[0,1])= \begin{cases}n^{\alpha} x, & x \in[0,1 / n] \\
n^{\alpha-1}, & \text { otherwise }\end{cases} \\
& \mu_{n}^{+}([0,1] \times[0, y])=\left\{\begin{array}{ll}
n^{\alpha} y, & y \in[0,1 / n] \\
n^{\alpha-1}, & \text { otherwise }
\end{array} .\right.
\end{aligned}
$$

Identical formulae are obtained for $\mu_{n}^{-}$. It follows, using (5.1), that

$$
\left\|A_{n}\right\|_{M}=2 n^{\alpha} .
$$

Now fix $\alpha \in(0,1)$. Observe that by (5.10) and (5.11), $\left\|A_{n}\right\|_{0, \infty}$ and $\left\|A_{n}\right\|_{J}$, which differ by a constant factor of 8 , both converge to 0 , whereas by (5.9) and (5.12), $\left|A_{n}\right|_{1, \infty}$ and $\left\|A_{n}\right\|_{M}$, which differ by a constant factor of 2 , both diverge to $+\infty$.

We turn to the interpretation of these two examples. Since the Minkowski norm dominates the Jordan norm, by Theorem 5.1, and the two norms cannot be equivalent, by Example 5.2, we obtain, by way of Lemma 4.3:

THEOREM 5.3. Span $(\mathcal{C})$ is not complete under the Jordan norm.

Observe that Example 5.1 completes the argument of Section 4 to similar effect: $\operatorname{span}(\mathcal{C})$ is not a closed subspace of $W^{1, p}$ for any $p \in[1, \infty]$.

Finally, observe that in view of Example 5.2 it cannot be true that $\|\cdot\|_{J}$ dominates $|\cdot|_{1, \infty}$ and that in view of Example 5.1 it cannot be true that $|\cdot|_{1, \infty}$ dominates $\|\cdot\|_{J}$.

\section{ONE PARAMETER SEMIGROUPS OF COPULAS}

We say $t \rightarrow C_{t}, t \geq 0$, is a one parameter semigroup if $C_{s+t}=C_{s} * C_{t}$ for all $s, t$.

Observe that $C_{t} * C_{0}=C_{0} * C_{t}=C_{t}$; setting $t=0$ we observe that $C_{0}$ is necessarily idempotent. Thus, if $C_{t}$ is a semigroup, $C_{0}$ is an idempotent which commutes with $C_{t}$ for all $t$.

If for some norm $\lim _{t \downarrow 0}\left\|C_{t}-C_{0}\right\|=0$ and the $*$ product is continuous in each place in the topology of the norm, then

$$
\lim _{t \downarrow t_{0}}\left\|C_{t}-C_{t_{0}}\right\|=\lim _{t \downarrow t_{0}}\left\|C_{t-t_{0}} * C_{t_{0}}-C_{t_{0}}\right\|=\left\|C_{0} * C_{t_{0}}-C_{t_{0}}\right\|=0 .
$$

That is, continuity at $t=0$ implies right continuity everywhere.

In fact, the existence of a right limit at $t=0$ implies right continuity everywhere on $(0, \infty)$, for reasonable norms:

THEOREM 6.1. Suppose $\|\cdot\|$ is a norm on a space containing span $(\mathcal{C})$, and suppose that the $*$ product on $\mathcal{C}$ is continuous in each place in the topology of the norm and also that norm convergence implies pointwise convergence. Let $t \rightarrow C_{t}$ be a semigroup of copulas, and suppose there is a function $E$ such that $\lim _{t \downarrow 0}\left\|C_{t}-E\right\|=0$. Then $E$ is idempotent, $E * C_{t}=C_{t} * E=C_{t}$ for all $t>0$ and $\lim _{t \downarrow t_{0}}\left\|C_{t}-C_{t_{0}}\right\|=0$ for all $t_{0}>0$.

PROOF. The proof is an adaptation of a classical proof to the case of copulas.

Observe first that since norm convergence implies pointwise convergence and $\mathcal{C}$ is compact in the uniform topology, norm convergence implies uniform convergence. Since the uniform limit of copulas is a copula, necessarily $E \in \mathcal{C}$. 
Since for $t>t_{0}>0, C_{t}=C_{t_{0}} * C_{t-t_{0}}=C_{t-t_{0}} * C_{t_{0}}$, onesided continuity of $*$ implies

$$
\begin{aligned}
& \lim _{t \downarrow t_{0}}\left\|C_{t}-C_{t_{0}} * E\right\|=0 \\
& \lim _{t \downarrow t_{0}}\left\|C_{t}-E * C_{t_{0}}\right\|=0 .
\end{aligned}
$$

Thus, right limits exist everywhere, and also $\lim _{t_{\downarrow} t_{0}} C_{t}=C_{t_{0}} * E=E * C_{t_{0}}$ for all $t_{0}>0$.

It remains to show that $C_{t_{0}}=C_{t_{0}} * E=E * C_{t_{0}}$. It suffices to show that $C_{t}$ is right continuous at a sequence of points $t_{n}$ converging to 0 , since if $C_{t_{n}}=C_{t_{n}} * E=E * C_{t_{n}}$ and $t>t_{n}$ then

$$
C_{t}=C_{t-t_{n}} * C_{t_{n}}=C_{t-t_{n}} * C_{t_{n}} * E=C_{t} * E .
$$

Fix $(x, y) \in[0,1]^{2}$, and define $f:[0, \infty) \rightarrow[0,1]$ via $f(t)=C_{t}(x, y)$. Observe that since norm convergence implies pointwise convergence, right $\operatorname{limits} \lim _{t \downarrow t_{0}} f(t)$ exist everywhere. It follows by a classical argument (an outline of the argument is given by Hille and Phillips [4,p. 282]) that the set $\mathcal{D}(x, y)$ of discontinuities of $f$ is countable. Now let $(x, y)$ vary over the rational pairs $(p, q) \in[0,1]^{2}$, and set $\mathcal{D}=\cup \mathcal{D}(p, q)$. Then $\mathcal{D}$ is countable. Since copulas are all Lipschitz continuous with Lipschitz constant 1, Darsow et al. [2], hence equicontinuous, it follows that

$$
\lim _{t \downarrow t_{0}} C_{t}=C_{t_{0}}
$$

pointwise for all $t_{0} \notin \mathcal{D}$. Since $(0, \infty) \backslash \mathcal{D}$ includes a sequence converging to 0 , we have right continuity everywhere.

That $E$ is idempotent now follows easily from the facts that $E * C_{t}=C_{t} * E=C_{t}$ for all $t>0$, that $\lim _{t \downarrow 0} C_{t}=E$ and that the $*$ product is continuous in each place.

Note that Theorem 6.1 requires only that the * product be continuous in each place and that it is the * product restricted to $\mathcal{C}$, rather than on all of span $(\mathcal{C})$, for which continuity is required. Accordingly, we can take any of $\|\cdot\|_{0, \infty},|\cdot|_{1, p}, p \in[1, \infty)$ or $\|\cdot\|_{M}$ for the norm in Theorem 6.1, since the $*$ product is continuous in each place with respect to each of these norms, and since for each norm convergence implies pointwise convergence. The theorem then states that a semigroup $C_{t}$ for which a right norm limit exists at $t=0$ is necessarily right continuous everywhere in the sense of the norm topology. We do not know whether we can take $\|\cdot\|_{J}$ or $|\cdot|_{1, \infty}$ for the norm in Theorem 6.1, since we do not know whether the * product, restricted to $\mathcal{C}$ is continuous in each place in the topology of these norms.

Note also that, if we are willing to assume that $\lim _{t \downarrow 0} C_{t}$ exists, there is no loss of generality in making the stronger assumption that $C_{t}$ is right continuous at $t=0$, since by Theorem 6.1, we can always redefine $C_{0}$ to be the $\operatorname{limit} E=\lim _{t \downarrow 0} C_{t}$ and still have a semigroup.

We address next the issue of whether measurability implies continuity for a one parameter semigroup of copulas. There is a classical argument that this is the case for one parameter semigroups in a Banach algebra, Hille et al. [4, p. 280].

In the following discussion, measurability of a function $f: R \rightarrow \mathcal{B}$ where $\mathcal{B}$ is a Banach space means "strong" measurability, that is, $f$ is measurable if for any compact subset $K \subset R$, $\left.f\right|_{K}$ is the limit pointwise almost everywhere of a sequence of measurable functions with finite range. A function with finite range is measurable if the inverse image of each point in the range is measurable. We will make use below of the following facts:

1. Strong measurability implies "weak" measurability; a function $f: R \rightarrow \mathcal{B}$ is weakly measurable if $t \rightarrow \nu(f(t))$ is a measurable scalar valued function for each $\nu \in \mathcal{B}^{*}$.

2. Strong and weak measurability are equivalent when the Banach space $\mathcal{B}$ is separable, Hille et al. $\left[4\right.$, p.73]. In this connection, recall that the spaces $W^{m, p}$ are separable for $p \in(1, \infty)$, Adams [1, Theorem 3.5]. 
3. (Approximation by continuous functions:) Let $\mathcal{L}^{p}(\Omega)$ denote the space of integrable $\mathcal{B}$ valued functions with domain $\Omega \subset R$; the set of continuous Banach space valued functions is dense in $\mathcal{L}^{p}(\Omega)$ for all $p \in[1, \infty)$, Hille et al. [4, p. 86].

Observe that if $f: R \rightarrow \mathcal{B}$ is right continuous, then it is measurable. To see this, let

$$
\mathcal{P}: a=t_{0}<t_{1}<\ldots<t_{n}=b
$$

denote a partition of a closed bounded interval $[a, b]$ and define $f^{\mathcal{P}}$ via

$$
f_{t}^{\mathcal{P}}= \begin{cases}\sum_{k=1}^{n} f_{t_{k}} I_{\left(t_{k-1}, t_{k}\right)}(t), & t \in[a, b) \\ f_{t}, & t=b\end{cases}
$$

where $I_{\left(t_{k-1}, t_{k}\right)}$ denotes the characteristic function of the interval $\left[t_{k-1}, t_{k}\right)$. Then for any sequence of partitions for which the maximum subinterval width $|\mathcal{P}|$ goes to zero, $\lim _{|\mathcal{P}| \rightarrow 0}\left\|f_{t}-f_{t}^{\mathcal{P}}\right\|=0$ for all $t$. Accordingly, if we make the assumption that a semigroup $t \rightarrow C_{t}$ is right continuous at $t=0$ in the sense of some norm, then by Theorem 6.1 and the preceding observation, $t \rightarrow C_{t}$ is measurable in the sense of that same norm.

The classical argument that measurability implies continuity uses the Banach algebra inequality. The proof of the following theorem follows the classical proof but replaces the Banach algebra inequality by a more restrictive condition.

THEOREM 6.2. Suppose $t \rightarrow C_{t}$ is a one parameter semigroup of copulas. Suppose also that $t \rightarrow C_{t}$ is measurable in the sense of a norm satisfying the following conditions:

(1) There is a number $M_{1}$ such that $\left\|C_{t}\right\| \leq M_{1}$ for all $t$, and

(2) There is a number $M_{2}$ such that for any $s, t$, and $u$

$$
\left\|C_{s} * C_{u}-C_{t} * C_{u}\right\| \leq M_{2}\left\|C_{s}-C_{t}\right\|
$$

Then $t \rightarrow C_{t}$ is continuous on $(0, \infty)$.

PROOF. Let $t>0$; we want to show that $\left\|C_{t+\eta}-C_{t}\right\| \rightarrow 0$ as $\eta \rightarrow 0$. Let $0<a<b<t$ and let $|\eta|<t-b$. Then for all $s \in[a, b]$ we have

$$
C_{t+\eta}-C_{t}=\left(C_{t+\eta-s}-C_{t-s}\right) * C_{s}=C_{s} *\left(C_{t+\eta-s}-C_{t-s}\right) .
$$

The last term in this expression is constant, hence bounded and integrable, and we have

$$
\begin{aligned}
(b-a)\left\|C_{t+\eta}-C_{t}\right\| & =\left\|\int_{a}^{b}\left(C_{t+\eta-s}-C_{t-s}\right) * C_{s} d s\right\| \\
& \leq \int_{a}^{b}\left\|\left(C_{t+\eta-s}-C_{t-s}\right) * C_{s}\right\| d s \\
& \leq M_{2} \int_{t-b}^{t-a}\left\|C_{u+\eta}-C_{u}\right\| d u .
\end{aligned}
$$

Condition (1) guarantees that the integrand of the last integral above is bounded. By a result mentioned above, together with some standard arguments, we can find a sequence of uniformly bounded continuous functions $t \rightarrow g_{t}^{n}$ converging pointwise to the bounded measurable function $C$ almost everywhere on the compact interval $[0, t]$, that is, for almost all $s,\left\|C_{s}-g_{s}^{n}\right\| \rightarrow 0$ as $n \rightarrow \infty$. With $C$ replaced by $g^{n}$ the integral in the last term in (6.3) goes to zero as $\eta \rightarrow 0$. It follows, via the triangle inequality and the dominated convergence theorem, that

$$
\int_{t-b}^{t-a}\left\|C_{u+\eta}-C_{u}\right\| d u \rightarrow 0
$$

as $\eta \rightarrow 0$. This yields the desired result. 
We will show below that the Sobolev norm $|\cdot|_{1,1}$ satisfies conditions (1) and (2) of Theorem 6.2 , and that as a result measurability of a semigroup $t \rightarrow C_{t}$ of copulas in the sense of $W^{1, p}$, for $p \in[1, \infty)$, implies continuity of the semigroup in the sense of $W^{1, p}, p \in[1, \infty)$. We are unable to show that condition (2) is satisfied by the uniform norm $\|\cdot\|_{0, \infty}$. It is an open question whether a semigroup which is measurable in the sense of the uniform norm is necessarily continuous.

We establish the inequality (6.1) for $p=1$, with $M_{2}=2$. Observe first that

$$
\begin{aligned}
& \left\|\left(\left(C_{s}-C_{t}\right) * C_{u}\right)_{, 1}\right\|_{0,1}=\left\|\left(C_{u} *\left(C_{s}-C_{t}\right)\right)_{, 1}\right\|_{0,1} \leq\left|C_{s}-C_{t}\right|_{1,1} \\
& \left\|\left(\left(C_{s}-C_{t}\right) * C_{u}\right)_{, 2}\right\|_{0,1} \leq\left|C_{s}-C_{t}\right|_{1,1}
\end{aligned}
$$

by (4.3). It follows that

$$
\begin{aligned}
\left|\left(C_{s}-C_{t}\right) * C_{u}\right|_{1,1} & =\left\|\left(\left(C_{s}-C_{t}\right) * C_{u}\right)_{, 1}\right\|_{0,1}+\left\|\left(\left(C_{s}-C_{t}\right) * C_{u}\right)_{, 2}\right\|_{0,1} \\
& \leq 2\left|C_{s}-C_{t}\right|_{1,1} .
\end{aligned}
$$

This yields $(6.1)$ in the case of $|\cdot|_{1,1}$. Observe that since $[0,1]^{2}$ is compact, for $p>1$, the $W^{1, p}\left([0,1]^{2}\right)$ norm dominates the $W^{1,1}\left([0,1]^{2}\right)$ norm, so measurability in the sense of $W^{1, p}$ implies measurability in the sense of $W^{1,1}$. Accordingly, if $t \rightarrow C_{t}$ is measurable in the sense of $W^{1, p}$, it is measurable also in the sense of $W^{1,1}$, whence, by Theorem 6.2 , it is continuous in the sense of $W^{1,1}$. Since the topologies of $W^{1, p}$ restricted to $\mathcal{C}$ coincide for $p \in[1, \infty)$, Lemma 4.1 , we conclude that $t \rightarrow C_{t}$ is continuous in the sense of $W^{1, p}$ for all $p \in[1, \infty)$.

Thus, a one parameter semigroup of copulas which is measurable in the sense of $|\cdot|_{1, p}$ is necessarily continuous in the sense of $|\cdot|_{1, p}$, for $p \in[1, \infty)$.

COROLLARY 6.1. A semigroup $t \rightarrow C_{t}$ of copulas which is right continuous at $t=0$ in the uniform norm is continuous for all $t \in(0, \infty)$ in the norm $|\cdot|_{1, p}$ for all $p \in[1, \infty)$.

PROOF. Let $t \rightarrow C_{t}$ satisfy $\lim _{t \downarrow 0}\left\|C_{t}-C_{0}\right\|_{0, \infty}=0$. Then by Theorem $6.1, t \rightarrow C_{t}$ is right continuous everywhere in the sense of the uniform norm. If $\nu$ is a continuous linear functional on $W^{1, p}\left([0,1]^{2}\right), p \in(1, \infty), t \rightarrow \nu\left(C_{t}\right)$ is a right continuous real valued function, by Theorem 4.4 , hence measurable, by an observation above. Thus, $t \rightarrow C_{t}$ is weakly measurable in the sense of $W^{1, p}$. But weak and strong measurability are equivalent for the separable spaces $W^{1, p}$, $1 \leq p<\infty$, so, by Theorem $6.2, t \rightarrow C_{t}$ is continuous in the sense of $|\cdot|_{1, p}$ for all $t>0$.

One might reasonably expect that right continuity in the sense of the uniform norm $\|\cdot\|_{0, \infty}$ would imply measurability in the sense even of the Minkowski norm $\|\cdot\|_{M}$, since on heuristic grounds one expects reasonable functions to be measurable. Then right continuity at 0 in the uniform norm would imply continuity for all $t>0$ in the sense of $\|\cdot\|_{M}$, directly by the classical Banach algebra argument, without worrying about how to obtain (6.1) in the case of Sobolev norms. It is an open issue whether this is the case. Hille and Phillips raise the same issue in the related context of transition matrices of Markov processes with discrete (but not finite) range, [4, p. 635]. We point out that measurability of a Banach space valued function depends on the norm used, so that the copula valued functions $t \rightarrow C_{t}$ here of interest may be measurable or not, depending on what Banach space we take $\mathcal{C}$ to be a subset of.

We now turn to the issue of analyticity of a semigroup $C_{t}$.

THEOREM 6.3. Let $C_{t}$ be a semigroup in $\mathcal{C}$, and suppose that $\lim _{t \downarrow 0}\left\|C_{t}-E\right\|_{M}=0$, where $E=C_{0}$. Then there is an $A \in \operatorname{span}(\mathcal{C})$, satisfying $A * E=E * A=A$, such that

$$
C_{t}=e^{A t}
$$

for all $t \geq 0$, where $e^{A t}$ is defined by its series representation, with the convention $A^{0}=E$.

This is a classical result for Banach algebras; for a proof, see Hille et al. [4].

It is an open question whether the generator $A$ of the semigroup $C_{t}$ in Theorem 6.3 is required to have the form $A=C-E$, where $C$ and $E$ are copulas, $E$ is idempotent, and $E C=C E=C$. 
It follows from Theorem 6.3 and some other elementary arguments that a semigroup $C_{t}$ of copulas possesses an exponential representation $C_{t}=e^{A t}$ for some $A \in \operatorname{span}(\mathcal{C})$ if and only if $\lim _{t \downarrow 0}\left\|C_{t}-E\right\|_{M}=0$, where $E=C_{0}$. This says roughly that the Minkowski norm is a natural one for one parameter semigroups with generators in span $(\mathcal{C})$.

It is by no means true that all one parameter semigroups of copulas possess exponential representations. We next exhibit a one parameter semigroup $C_{t}$ which is right continuous at 0 in the sense of $|\cdot|_{1, p}$ for $p \in[1, \infty)$ but not in the sense of $|\cdot|_{1, \infty}$ and hence, by Theorem 4.6, also not in the sense of $\|\cdot\|_{M}$. By Corollary 6.2 , therefore, the semigroup does not possess an exponential representation.

EXAMPLE 6.1. It was shown in Darsow et al. [2] that the copulas of a Wiener process have the form

$$
C_{s, t}(x, y)=\int_{0}^{x} G\left(\frac{\sqrt{t} G^{-1}(y)-\sqrt{s} G^{-1}(u)}{\sqrt{t-s}}\right) d u
$$

where $s<t$ and

$$
G(x)=\frac{1}{\sqrt{2 \pi}} \int_{-\infty}^{x} e^{-u^{2} / 2} d u .
$$

Since the Wiener process is a Markov process, we have, for $s<u<t$,

$$
C_{s ; u} * C_{u ; t}=C_{s ; t} .
$$

Equation (6.4) implies the following scaling property: if $r>0$ and $s<t$ then

$$
C_{s / r ; t / r}=C_{s ; t}
$$

It follows from (6.5) and (6.6) that the family of copulas $B_{t}$ defined by

$$
B_{t}=C_{1 ; e^{t}}
$$

where $C_{s ; t}$ is given by (6.4), has the semigroup property for $t>0$, as is readily verified. (We remark that it is also possible to go backwards: if $B_{t}^{\prime}$ is any one parameter semigroup of copulas and we define $C_{s ; t}^{\prime}=B_{\ln t / s}^{\prime}$ for $s<t$, then the family $C_{s ; t}^{\prime}$ satisfies the Markov condition (6.5) and also the scaling property (6.6) of a Wiener process.)

We will show that $\lim _{t \downarrow 0}\left\|B_{t}-M\right\|_{1, p}=0$ for $p \in[1, \infty)$ but not for $p=\infty$.

From the definition, we have

$$
B_{t}(x, y)=\int_{0}^{x} G\left(\frac{e^{t / 2} G^{-1}(y)-G^{-1}(u)}{\sqrt{e^{t}-1}}\right) d u .
$$

It follows, after making a substitution in the integral, that

$$
B_{t}(G(x), G(y))=\frac{1}{\sqrt{2 \pi}} \int_{-\infty}^{x} G\left(\frac{e^{t / 2} y-u}{\sqrt{e^{t}-1}}\right) e^{-u^{2} / 2} d u
$$

Since

$$
\lim _{t \downarrow 0} G\left(\frac{e^{t / 2} y-u}{\sqrt{e^{t}-1}}\right)= \begin{cases}\sqrt{2 \pi}, & y \geq u \\ 0, & y<u\end{cases}
$$

we have

$$
\begin{aligned}
\lim _{t \downarrow 0} B_{t}(G(x), G(y)) & =\int_{-\infty}^{x} \chi_{(-\infty, y]}(u) e^{-u^{2} / 2} d u \\
& =\int_{-\infty}^{\min \{x, y\}} e^{-u^{2} / 2} d u \\
& =G(\min \{x, y\}) \\
& =\min \{G(x), G(y)\}) .
\end{aligned}
$$


Hence, $\lim _{t \downarrow 0} B_{t}=M$ pointwise, and since $\mathcal{C}$ is compact in the uniform topology, also uniformly. By similar arguments, $\lim _{t \downarrow 0} B_{t, 1}=M_{, 1}$ pointwise a.e. and $\lim _{t \downarrow 0} B_{t, 2}=M_{, 2}$ pointwise a.e. From the foregoing results and the dominated convergence theorem, we obtain

$$
\lim _{t \downarrow 0}\left\|B_{t}-M\right\|_{1, p}=0
$$

for $p \in[1, \infty)$. The result does not hold for $p=\infty$, however; the partial derivatives $B_{t, 1}$ and $B_{t, 2}$ are continuous for all $t>0$, but $M_{, 1}$ and $M_{, 2}$ are not continuous functions, hence not the uniform limit of continuous functions.

That $B_{t}$ possesses no exponential representation now follows from an observation made above (see discussion following Theorem 6.3). Observe that if $B_{t}$ possessed an exponential representation $B_{t}=e^{A t}$, then it would be true that $\lim _{t \downarrow 0} d B_{t} / d t=A$. It can be verified directly that the semigroup $B_{t}$ of this example does not possess an exponential representation, since the latter limit is 0 except when $x=y$, when it is infinite.

\section{DISCUSSION.}

We conclude with some remarks about compactness. Recall that $\mathcal{C}$ is a compact subset of $W^{0, \infty}$ and also that the $*$ product fails to be jointly continuous in the topology of uniform convergence. The question is whether $\mathcal{C}$ itself or any useful subset is compact under any of the other norms addressed. It is easy to verify that if $\|\cdot\|$ is any norm which dominates the uniform norm, and $\mathcal{C}$ is compact under the topology of $\|\cdot\|$, then necessarily the * product on $\mathcal{C}$ is not jointly continuous in the topology of $\|\cdot\|$. This implies that $\mathcal{C}$ is not a compact subset of $W^{1, p}$ for $p \in[1, \infty)$, since the $*$ product is jointly continuous on $\mathcal{C}$ with respect to the norms of these Sobolev spaces, Theorem 4.2. More generally, this seems to imply that we cannot obtain both compactness of $\mathcal{C}$ and joint continuity of the * product in a single topology. We thought at one time that it might be possible to find a topology under which the group $\mathcal{G} \subset \mathcal{C}$ of invertible copulas was a compact group. But this seems unlikely, for the following reasons: $\mathcal{G}$ is dense in $\mathcal{C}$ in the uniform topology, Darsow et al. [2], and thus is dense in the topology of any norm which is dominated by the uniform norm. Compactness fails because $\mathcal{G}$ is not closed. On the other hand, if $\|\cdot\|$ dominates the uniform norm, and $C_{n}$ is a sequence of invertible copulas converging uniformly to a noninvertible copula, say $P$, then if $\mathcal{G}$ were compact in the topology of $\|\cdot\|$, there would be a subsequence converging to an element of $\mathcal{G}$, contradiction, since then the subsequence would necessarily also converge uniformly to the element of $\mathcal{G}$. A norm with respect to which $\mathcal{G}$ was compact could accordingly neither dominate nor be dominated by the uniform norm.

We consider it unlikely that any norms in which the * product behaves well will be found to possess any useful compactness properties.

\section{REFERENCES}

[1] R.A. Adams, Sobolev Spaces (Academic Press, New York, 1975)

[2] W.F. Darsow, B. Nguyen and E.T. Olsen, "Copulas and Markov Processes," Ill. J. Math. 36:600-642 (1992)

[3] P.R. Halmos, Measure Theory (Van Nostrand, Princeton, 1950)

[4] E. Hille and R.S. Phillips, Functional Analysis and Semi-groups

(American Mathematical Society, New York, 1957)

[5] W.Rudin, Functional Analysis (McGraw-Hill, New York, 1973)

[6] A. Sklar, "Fonctions de répartition à n dimensions et leur marges," Publ. Inst. Statist. Univ. Paris 8:229-231 (1959)

[7] A. Sklar, "Random Variables, Joint Distribution Functions and Copulas," Kybernetika 9:449-460 (1973) 


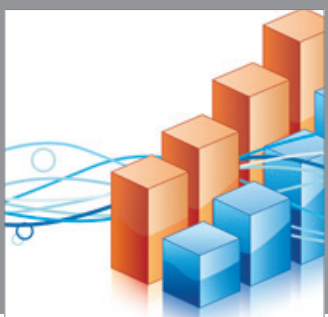

Advances in

Operations Research

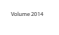

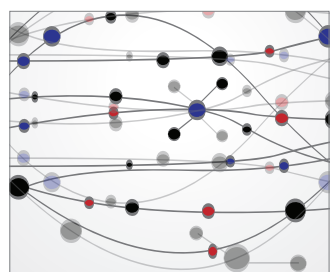

\section{The Scientific} World Journal
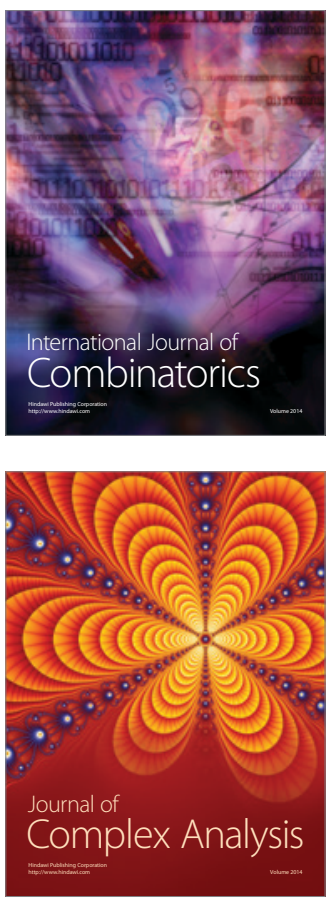

International Journal of

Mathematics and

Mathematical

Sciences
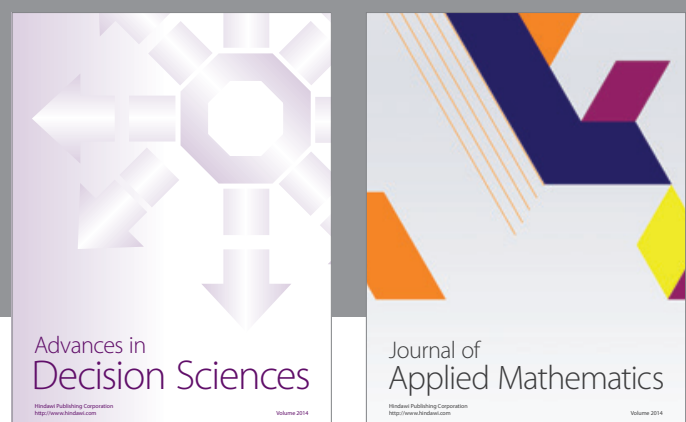

Journal of

Applied Mathematics
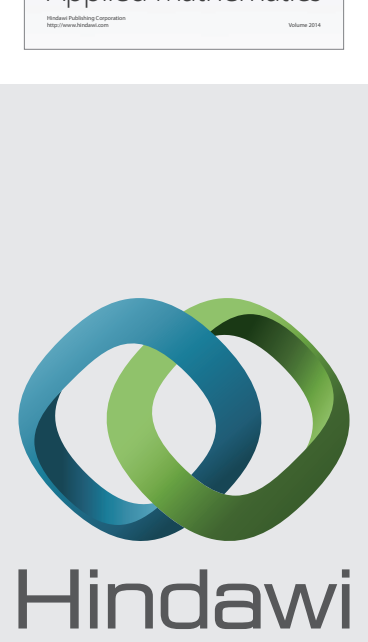

Submit your manuscripts at http://www.hindawi.com
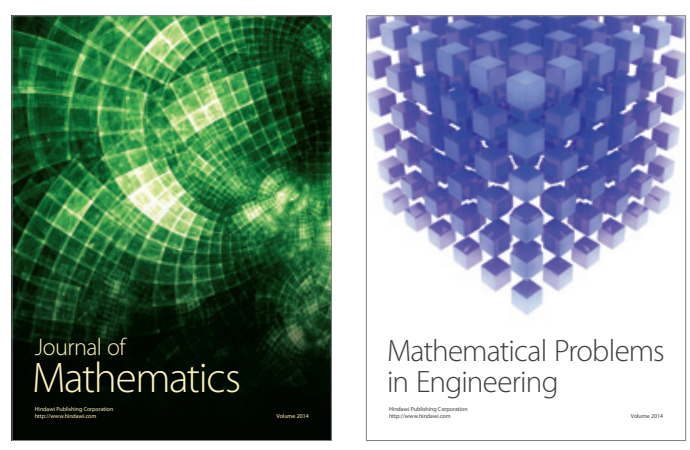

Mathematical Problems in Engineering
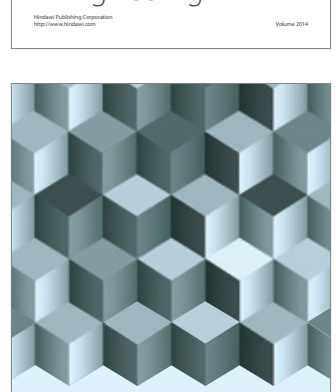

Journal of

Function Spaces
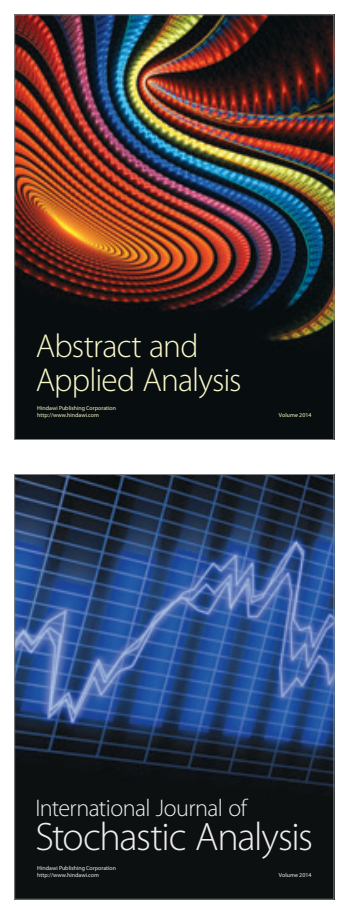

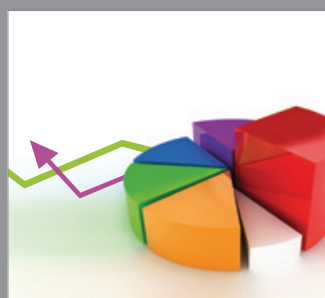

ournal of

Probability and Statistics

Promensencen
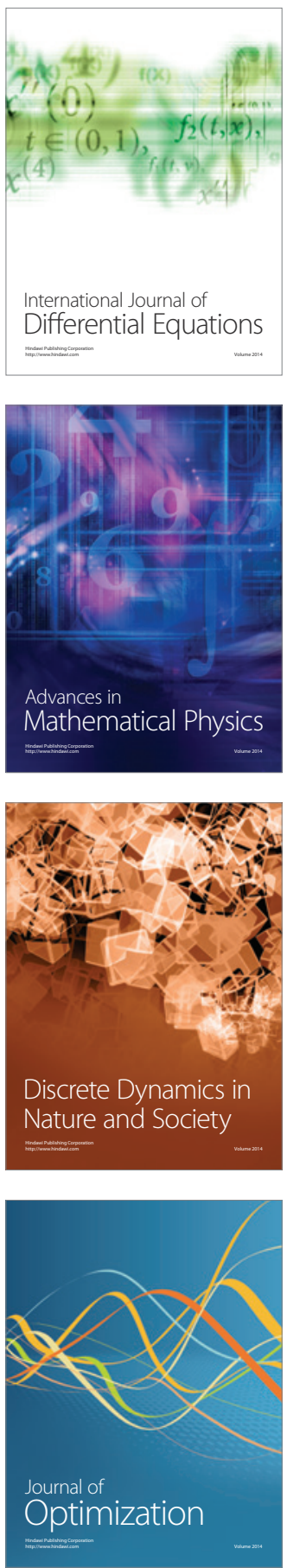